Research Article

\title{
Propagation of Transverse Wave for the Piezoelectric Radial Phononic Crystal Annular Plate in a Fibonacci Order
}

\author{
Wei Liu $\mathbb{D I}^{1}$ and Guangbin Yu $\mathbb{1 D}^{2}$ \\ ${ }^{1}$ School of Traffic and Transportation, Northeast Forestry University, Harbin 150040, China \\ ${ }^{2}$ School of Mechatronics Engineering, Harbin Institute of Technology, Harbin 150001, China \\ Correspondence should be addressed to Guangbin Yu; yugbhit@163.com
}

Received 6 May 2021; Revised 20 July 2021; Accepted 22 July 2021; Published 31 July 2021

Academic Editor: Ömer Cívalek

Copyright (c) 2021 Wei Liu and Guangbin Yu. This is an open access article distributed under the Creative Commons Attribution License, which permits unrestricted use, distribution, and reproduction in any medium, provided the original work is properly cited.

\begin{abstract}
Based on the previous conventional phononic crystal (PC) structures infinitely periodic in Cartesian coordinates, this paper addresses a new radial phononic crystal annular plate (RPCAP) modeled in a Fibonacci order along the radial direction. In this study, piezoelectric material PZT4 is simultaneously inserted into this RPCAP model to investigate the stop band behaviors. In order to clearly show the transmission characteristics of transverse wave, in cylindrical coordinates, the transfer matrix is deduced through combining the general solutions, piezoelectric governing equations, and continuity conditions. Compared with conventional PC structures, transmission response of transverse vibration for the Fibonacci RPCAP model is calculated theoretically to analyze the stop band phenomenon. Finite element simulation method (FEM) is conducted here to verify the theoretical results. The results show that the Fibonacci RPCAP model presents two newly emerging resonance frequencies while the radial periodic order is disorganized. To thoroughly understand the RPCAP, the effects of structural parameters, material parameters, and piezoelectric parameters on the stop band are discussed in detail. The proposed Fibonacci RPCAP can be employed in many engineering applications, such as in rotating parts which are often coupled with transverse vibration (like gear driving systems).
\end{abstract}

\section{Introduction}

PC is a new type of artificial periodic composite structures. These composite structures exhibit unique dynamic behaviors, such as wave filters which can make some elastic waves completely blocked within a certain frequency range called the "stop band." Accordingly, extensive significant efforts have been devoted to the study of various PC structures in an attempt to stop the elastic wave propagation through properly designing these structures. Asiri et al. [1] developed a class of active PC structures consisting of two alternating homogeneous materials and piezoelectric materials to demonstrate the unique filtering capabilities. Their experimental results show that the designed stop band can be used to control the wave propagation from the gearbox to the airframe within the low frequency range. Wang et al. [2] constructed a novelty PC curved beam model to investigate the in-plane elastic wave band by utilizing the transfer matrix method. The obtained radial and tangential stop bands are verified by the finite element software. Through employing surface effect and Timoshenko beam theory, Qian et al. [3] proposed a piezoelectric PC nanobeam to analyze the electromechanical coupling band behaviors. The effects of electromechanical coupling fields, residual surface stresses, and geometric parameters on stop bands are discussed in detail. Yao et al. [4] applied the finite element method to calculate the elastic wave stop band characteristics of topological PC plates with local resonators. Yu et al. [5] investigated the complex flexural stop band characteristics of PC pipe system conveying fluid. And, the stop band properties were revealed deeply by Bragg scattering and locally resonant mechanisms using transfer matrix. Mazzotti et al. [6] made a further analysis for the band behavior of leaky Bloch waves in 2D PC plates using the hybrid finite 
element-plane wave expansion method. Chen et al. [7] emphasized on the active control of flexural wave of piezoelectric PC beam with staggered periodic properties. The effect of the degree of interlacing and negative capacitance circuits on the stop band was evaluated. Goto et al. [8] numerically calculated the Bragg scattering bands and forced responses of PC rod via the wave-based higher-order rod model and wave finite element method. The experimental test is performed to validate the numerical results. Zak et al. [9] investigated the longitudinal, flexural, and torsional wave band behaviors of a three-dimensional isotropic PC beam to achieve the vibroacoustic isolation purposes. Guo et al. [10] considered a kind of one-dimensional piezoelectric PC structures with imperfect interfaces, finding that the mechanically and dielectrically imperfect interface has evident influence on the stop bands.

In addition to the above conventional PC [1-10] structures with translational periodicity, currently research studies have been extensively expanded to a class of radial phononic crystal (RPC) structures with radial periodicity. In our previous work [11], the torsional band phenomenon of stress wave of generalized PC with radial periodicity has been confirmed, and the results show that the stop band phenomenon is similar to the existing PC. Simultaneously, the band behaviors of flexural wave propagating in the piezoelectric RPC was investigated. The effects of structural parameters and outer active control on the stop bands were analyzed [12]. In order to control the torsional wave propagation in the circular plate, Chai et al. [13] studied the Bragg scattering and locally resonant bands of torsional wave in the piezoelectric RPC, finding that the stop bands could be effectively varied through adjusting the external circuit parameters. Xu et al. [14] employed the transfer matrix method to analyze the low-frequency phononic bands of a two-dimensional arc-shaped PC in cylindrical coordinates. Similarly, by introducing specially designed acoustic metamaterials, Torrent et al. [15-17] made a comprehensive research for the radial sonic crystal to reveal the stop band mechanism.

To the authors' knowledge, the existing PC studies are mainly about the specially designed structures with translational periodicity or radial periodicity while research studies on Fibonacci RPC are rare. Actually, compared with periodic structures, the Fibonacci (nonperiodic) structures can exhibit many unique dynamic behaviors. In our earlier work, stop band of torsional wave for the Fibonacci RPC [18] has been primitively investigated. However, few studies on transverse wave stop band can be found. This is the first time that piezoelectric material PZT4 is introduced into the Fibonacci RPCAP to achieve the stop band of transverse wave.

In this paper, a Fibonacci RPCAP including piezoelectric material is proposed to analyze the stop band behaviors by employing the transfer matrix method. Stop bands calculated by Fibonacci RPCAP are compared with those obtained by periodic RPCAP. Afterwards, finite element simulation is carried out to verify the theoretical results. Finally, structural parameters and piezoelectric effects on the stop band are discussed in detail. The emphasis of this study is to reveal the band behaviors of Fibonacci RPCAP which is extremely significant for the vibration reduction of rotor machinery structures coupled with transverse vibration.

\section{Theoretical Basis of Piezoelectric Fibonacci RPCAP}

2.1. Fibonacci RPCAP Model. Consider a Fibonacci RPCAP model consisting of two different alternating materials as depicted in Figure 1. Figure 1(a) shows a basic model, and Figure 1(b) shows a quarter model. The RPCAP mode is arranged in the form of Fibonacci order constructed as ABAABABA type. Here, the first material is selected as resin and the second material is selected as PZT4. The piezoelectric material PZT4 polarized along the thickness direction is inserted into the Fibonacci RPCAP model. For this Fibonacci RPCAP model, inner radius and outer radius are $r_{1}$ and $r_{2}$. Radial lengths of the two materials are $a_{1}$ and $a_{2}$. Additionally, thickness of the resin is consistent with PZT4. The thickness is $h$.

\subsection{Governing Equation of Transverse Vibration}

2.2.1. Homogeneous Governing Equation. In cylindrical coordinates, governing equations of transverse vibration for the homogeneous material are defined as [12]

$$
\begin{gathered}
\frac{\partial M_{r}}{\partial r}+\frac{M_{r}-M_{\theta}}{r}=Q_{r}, \\
\frac{Q_{r}}{r}+\frac{\partial Q_{r}}{\partial r}+q=\rho h \frac{\partial^{2} w}{\partial t^{2}}, \\
M_{r}=\int_{-(h / 2)}^{h / 2}\left(T_{r} \mathrm{~d} z\right) z, \\
M_{\theta}=\int_{-(h / 2)}^{h / 2}\left(T_{\theta} \mathrm{d} z\right) z,
\end{gathered}
$$

where $M_{r}, M_{\theta}$ denote the radial and circumferential bending moments, $Q_{r}$ is the transverse shear, $q$ is the outer force (for free vibration, $q=0$ ), $r$ is the radial direction, $\theta$ is the circumferential direction, $z$ is the axle direction, and $w$ is the transverse displacement.

Since the transverse vibration is axisymmetric, the stress can be expressed as

$$
\begin{aligned}
& T_{r}=\frac{E}{1-\sigma^{2}}\left(S_{r}+\sigma S_{\theta}\right), \\
& T_{\theta}=\frac{E}{1-\sigma^{2}}\left(\sigma S_{r}+S_{\theta}\right),
\end{aligned}
$$

where $T_{r}$ is the radial stress, $T_{\theta}$ is the circumferential stress, $E$ is Young's modulus, and $\sigma$ is Poisson's coefficient.

The expressions of strain and transverse deformation are related by 


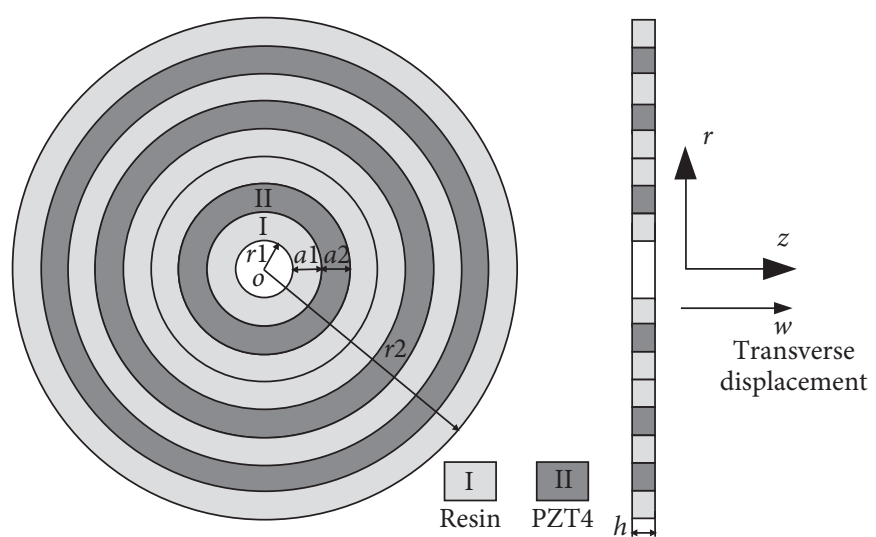

(a)

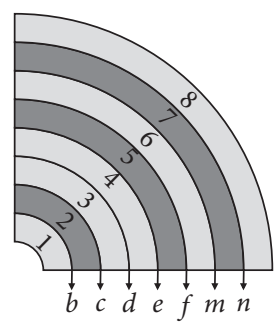

(b)

FIgURE 1: Schematic diagram of Fibonacci RPCAP model: (a) basic model; (b) a quarter model.

$$
\left\{\begin{array}{l}
S_{r}=-z \frac{\partial^{2} w}{\partial r^{2}}, \\
S_{\theta}=-\frac{z}{r} \frac{\partial w}{\partial r},
\end{array}\right.
$$

where $S_{r}, S_{\theta}$ are the radial and circumferential strains.

In this case, combining equations (1)-(7) reduces to

$$
\left(\frac{\partial^{2}}{\partial r^{2}}+\frac{1}{r} \frac{\partial}{\partial r}\right)\left(\frac{\partial^{2}}{\partial r^{2}}+\frac{1}{r} \frac{\partial}{\partial r}\right) W-k^{4} W=0
$$

where $W$ is the transverse deformation and $k$ is the wavenumber $\left(k^{4}=(\rho h / D)(2 \pi f)^{2}\right)$. Transverse bending stiffness is $D=E h^{3} / 12\left(1-\sigma^{2}\right) . f$ is the frequency.

According to equation (8), the following general solution is generated:

$$
W=A_{1} J_{0}(k r)+A_{2} Y_{0}(k r)+A_{3} I_{0}(k r)+A_{4} K_{0}(k r),
$$

where $A_{1}, A_{2}, A_{3}, A_{4}$ are the constants which can be determined by the boundaries, $J_{0}(k r), Y_{0}(k r)$ are the Bessel functions of the first and second kinds, and $I_{0}(k r), K_{0}(k r)$ are the modified Bessel functions of the first and second kinds, respectively.

2.2.2. Piezoelectric Governing Equation. Since polarization direction of piezoelectric material PZT4 is along $Z$-axis, the $g$ type piezoelectric governing equations are given by [12]

$$
\begin{aligned}
& S_{r}=s_{11}^{D} T_{r}+s_{12}^{D} T_{\theta}+g_{31} D_{z}, \\
& S_{\theta}=s_{12}^{D} T_{r}+s_{12}^{D} T_{\theta}+g_{31} D_{z}, \\
& E_{z}=-g_{31} T_{r}-g_{31} T_{\theta}+\beta_{33}^{T} D_{z} .
\end{aligned}
$$

Here equations (10)-(12) can be rearranged as

$$
\begin{aligned}
& T_{r}=\frac{1}{s_{11}^{D}\left(1-\sigma^{2}\right)} S_{r}+\frac{\sigma}{s_{11}^{D}\left(1-\sigma^{2}\right)} S_{\theta}-\frac{g_{31}}{s_{11}^{D}(1-\sigma)} D_{z}, \\
& T_{\theta}=\frac{\sigma}{s_{11}^{D}\left(1-\sigma^{2}\right)} S_{r}+\frac{1}{s_{11}^{D}\left(1-\sigma^{2}\right)} S_{\theta}-\frac{g_{31}}{s_{11}^{D}(1-\sigma)} D_{z}, \\
& E_{z}=\frac{g_{31} z}{s_{11}^{D}(1-\sigma)}\left(\frac{\partial^{2} w}{\partial r^{2}}+\frac{1}{r} \frac{\partial w}{\partial r}\right)+\bar{\beta}_{33} D_{z},
\end{aligned}
$$

where $\sigma=-\left(s_{12}^{D} / s_{11}^{D}\right)$.

Similar to homogeneous material, substituting equations (13) and (14) into equations (3) and (4), expression of transverse stress in equation (1) is generated by a simple derivation:

$$
Q_{r}=\frac{-h^{3}}{12 s_{11}^{D}\left(1-\sigma^{2}\right)} \frac{\partial}{\partial r}\left(\frac{\partial^{2} w}{\partial r^{2}}+\frac{1}{r} \frac{\partial w}{\partial r}\right)-\frac{g_{31} h^{2}}{4 s_{11}^{D}(1-\sigma)} \frac{\partial D_{z}}{\partial r}
$$

Transverse vibration equation with no load case can be described as

$$
\frac{Q_{r}}{r}+\frac{\partial Q_{r}}{\partial r}=\rho h \frac{\partial^{2} w}{\partial t^{2}}
$$

Integration can be conducted for piezoelectric constitutive equation $(15)$ in the frequency interval $[0, h / 2]$ from which one obtains

$$
D_{z}=\frac{2}{h \bar{\beta}_{33}} V-\frac{g_{31} h}{4 s_{11}^{D}(1-\sigma) \bar{\beta}_{33}}\left(\frac{\partial^{2} w}{\partial r^{2}}+\frac{1}{r} \frac{\partial w}{\partial r}\right) .
$$

The electric field is $E_{z}=0$ which corresponds to the electrical short circuit case, and then the charge satisfies the relation $D_{z} \neq 0$. Substituting equations (16) and (18) into equation (17) leads to

$$
\left(\frac{\partial^{2}}{\partial r^{2}}+\frac{1}{r} \frac{\partial}{\partial r}\right)\left(\frac{\partial^{2}}{\partial r^{2}}+\frac{1}{r} \frac{\partial}{\partial r}\right) W+\frac{\rho}{D} h=0
$$


For the piezoelectric material PZT4, general solution of transverse vibration is generated from equation (19):

$$
W=A_{1} J_{0}(\bar{k} r)+A_{2} Y_{0}(\bar{k} r)+A_{3} I_{0}(\bar{k} r)+A_{4} K_{0}(\bar{k} r) .
$$

Therefore, bending stiffness of piezoelectric material PZT4 for electrical short circuit case can be calculated as

$$
D^{E}=\frac{h^{3}}{12 s_{11}^{D}\left(1-\sigma^{2}\right)}-\frac{h^{3} g_{31}^{2}}{16\left(s_{11}^{D}\right)^{2}(1-\sigma)^{2} \bar{\beta}_{33}}=\frac{h^{3}}{12 s_{11}^{D}(1-\sigma)}\left(\frac{1}{1+\sigma}-\frac{3}{8} k_{p}^{2}\right)
$$

Similarly, while the charge is $D_{z}=0$ which corresponds to the electrical open circuit case, the electric field satisfies $E_{z} \neq 0$. Bending stiffness of piezoelectric material PZT4 for electrical open circuit case can be simplified as

$$
D^{D}=\frac{h^{3}}{12 s_{11}^{D}\left(1-\sigma^{2}\right)} \text {. }
$$

2.3. Transfer Matrix. In this section, transfer matrix of transverse vibration for piezoelectric Fibonacci RPCAP is deduced in detail. The obtained transfer matrix in cylindrical coordinates is helpful for calculating the stop band described in the transmission response curves.

Consider a piezoelectric Fibonacci RPCAP with eight layers corresponding to nonperiodic model ABAABABA, as depicted in Figure 1. Along the radial direction, segments of piezoelectric Fibonacci RPCAP are divided into seven interfaces marked as $b, c, d, e, f, m$, and $n$. The connections between the two materials are adopted for mechanical connection. To highlight the essence, here, connection types are treated as ideal. For the resin material propagating in 1, 3, 4, 6, 8 layers described in Figure 1(b), Young's modulus, density, and Poisson's coefficient are $E_{1}, \rho_{1}$, and $\sigma_{1}$. Similarly, for piezoelectric material PZT4 propagating in 2, 5, 7 layers, they are $E_{2}, \rho_{2}$, and $\sigma_{2}$. Wavenumbers of resin and PZT4 can be expressed as $k_{1}^{4}=\left(\rho_{1} h_{1} / D_{1}\right)(2 \pi f)^{2}$ and $k_{2}^{4}=\left(\rho_{2} h_{2} / D_{2}\right)(2 \pi f)^{2}$. The stiffness can be calculated as $D_{1}=E_{1} h_{1}^{3} / 12\left(1-\sigma_{1}^{2}\right)$ and $D_{2}=E_{2} h_{2}^{3} / 12\left(1-\sigma_{2}^{2}\right)$.

While transverse wave propagates in piezoelectric Fibonacci RPCAP, the physical parameters such as displacement, angle, moment, and shear force should satisfy the following continuity condition at the interface of different materials. With regard to resin material, specific expressions of these physical parameters can be written as

$$
\begin{aligned}
& W_{1}\left(r^{\prime}\right)=A_{11} J_{0}\left(k_{1} r^{\prime}\right)+B_{11} Y_{0}\left(k_{1} r^{\prime}\right)+C_{11} I_{0}\left(k_{1} r^{\prime}\right)+D_{11} K_{0}\left(k_{1} r^{\prime}\right), \\
& \begin{array}{l}
\frac{\partial W}{\partial r^{\prime}}\left(r^{\prime}\right)=-k_{1} A_{11} J_{1}\left(k_{1} r^{\prime}\right)-k_{1} B_{11} Y_{1}\left(k_{1} r^{\prime}\right)+k_{1} C_{11} I_{1}\left(k_{1} r^{\prime}\right)-k_{1} D_{11} K_{1}\left(k_{1} r^{\prime}\right), \\
M_{1}\left(r^{\prime}\right)=D_{1}\left\{\begin{array}{l}
A_{11}\left[\frac{k_{1}}{r^{\prime}} J_{1}\left(k_{1} r^{\prime}\right)-\frac{\sigma_{1} k_{1}}{r^{\prime}} J_{1}\left(k_{1} r^{\prime}\right)-k_{1}^{2} J_{0}\left(k_{1} r^{\prime}\right)\right]+B_{11}\left[\frac{k_{1}}{r^{\prime}} Y_{1}\left(k_{1} r^{\prime}\right)-\frac{\sigma_{1} k_{1}}{r^{\prime}} Y_{1}\left(k_{1} r^{\prime}\right)-k_{1}^{2} Y_{0}\left(k_{1} r^{\prime}\right)\right] \\
+C_{11}\left[k_{1}^{2} I_{0}\left(k_{1} r^{\prime}\right)-\frac{k_{1}}{r^{\prime}} I_{1}\left(k_{1} r^{\prime}\right)+\frac{\sigma_{1} k_{1}}{r^{\prime}} I_{1}\left(k_{1} r^{\prime}\right)\right]+D_{11}\left[k_{1}^{2} K_{0}\left(k_{1} r^{\prime}\right)+\frac{k_{1}}{r^{\prime}} K_{1}\left(k_{1} r^{\prime}\right)-\frac{\sigma_{1} k_{1}}{r^{\prime}} K_{1}\left(k_{1} r^{\prime}\right)\right]
\end{array}\right\}, \\
Q_{1}\left(r^{\prime}\right)=D_{1}\left\{A_{1} k_{1}^{3} J_{1}\left(k_{1} r^{\prime}\right)+B_{11} k_{1}^{3} Y_{1}\left(k_{1} r^{\prime}\right)+C_{11} k_{1}^{3} I_{1}\left(k_{1} r^{\prime}\right)-D_{11} k_{1}^{3} K_{1}\left(k_{1} r^{\prime}\right)\right\} .
\end{array}
\end{aligned}
$$

For the piezoelectric material PZT4, these physical parameters are 


$$
\begin{aligned}
& W_{2}\left(r^{\prime}\right)=A_{22} J_{0}\left(k_{2} r^{\prime}\right)+B_{22} Y_{0}\left(k_{2} r^{\prime}\right)+C_{22} I_{0}\left(k_{2} r^{\prime}\right)+D_{22} K_{0}\left(k_{2} r^{\prime}\right), \\
& \frac{\partial W}{\partial r_{2}}\left(r^{\prime}\right)=-k_{22} A_{2} J_{1}\left(k_{2} r^{\prime}\right)-k_{22} B_{2} Y_{1}\left(k_{2} r^{\prime}\right)+k_{2} C_{22} I_{1}\left(k_{2} r^{\prime}\right)-k_{2} D_{22} K_{1}\left(k_{2} r^{\prime}\right), \\
& M_{2}\left(r^{\prime}\right)=D_{2}\left\{\begin{array}{l}
A_{22}\left[\frac{k_{2}}{r^{\prime}} J_{1}\left(k_{2} r^{\prime}\right)-\frac{\sigma_{2} k_{2}}{r^{\prime}} J_{1}\left(k_{2} r^{\prime}\right)-k_{2}^{2} J_{0}\left(k_{2} r^{\prime}\right)\right]+B_{22}\left[\frac{k_{2}}{r^{\prime}} Y_{1}\left(k_{2} r^{\prime}\right)-\frac{\sigma_{2} k_{2}}{r^{\prime}} Y_{1}\left(k_{2} r^{\prime}\right)-k_{2}^{2} Y_{0}\left(k_{2} r^{\prime}\right)\right] \\
+C_{22}\left[k_{2}^{2} I_{0}\left(k_{2} r^{\prime}\right)-\frac{k_{2}}{r^{\prime}} I_{1}\left(k_{2} r^{\prime}\right)+\frac{\sigma_{2} k_{2}}{r^{\prime}} I_{1}\left(k_{2} r^{\prime}\right)\right]+D_{22}\left[k_{2}^{2} K_{0}\left(k_{2} r^{\prime}\right)+\frac{k_{2}}{r^{\prime}} K_{1}\left(k_{2} r^{\prime}\right)-\frac{\sigma_{2} k_{2}}{r^{\prime}} K_{1}\left(k_{2} r^{\prime}\right)\right]
\end{array}\right\}, \\
& Q_{2}\left(r^{\prime}\right)=D_{2}\left\{A_{22} k_{2}^{3} J_{1}\left(k_{2} r^{\prime}\right)+B_{22} k_{2}^{3} Y_{1}\left(k_{2} r^{\prime}\right)+C_{22} k_{2}^{3} I_{1}\left(k_{2} r^{\prime}\right)-D_{22} k_{2}^{3} K_{1}\left(k_{2} r^{\prime}\right)\right\} .
\end{aligned}
$$

According to the continuity condition at the interface $r_{b}=r 1+a 1$, the relationship is

$$
\begin{aligned}
& W_{n 1}\left(r_{b}\right)=W_{n 2}\left(r_{b}\right), \\
& \frac{\partial W}{\partial r_{n 1}}\left(r_{b}\right)=\frac{\partial W}{\partial r_{n 2}}\left(r_{b}\right) \text {, } \\
& M_{n 1}\left(r_{b}\right)=M_{n 2}\left(r_{b}\right) \text {, } \\
& Q_{n 1}\left(r_{b}\right)=Q_{n 2}\left(r_{b}\right) \text {. }
\end{aligned}
$$

Here, equation (25) can be rewritten in a matrix form:

$$
\mathrm{K}_{1} \psi_{1}=\mathrm{H}_{1} \psi_{2} \text {, }
$$

where $\psi_{1}=\left[\begin{array}{llll}A_{11} & B_{11} & C_{11} & D_{11}\end{array}\right]^{T}$ and $\psi_{2}=\left[\begin{array}{lll}A_{22} & B_{22} & C_{22}\end{array}\right.$ $\left.D_{22}\right]^{T}$.

Similarly, according to the continuity condition at the interface $r_{c}=r 1+a 1+a 2$, one can obtain the following relationship:

$$
\begin{aligned}
& W_{n 1}\left(r_{c}\right)=W_{n 2}\left(r_{c}\right), \\
& \frac{\partial W}{\partial r_{n 1}}\left(r_{c}\right)=\frac{\partial W}{\partial r_{n 2}}\left(r_{c}\right), \\
& M_{n 1}\left(r_{c}\right)=M_{n 2}\left(r_{c}\right) \text {, } \\
& Q_{n 1}\left(r_{c}\right)=Q_{n 2}\left(r_{c}\right) .
\end{aligned}
$$

Then, equation (27) reduces to

$$
\mathbf{K}_{2} \psi_{3}=\mathbf{H}_{2} \psi_{2} .
$$

Combining equations (26) and (28), one has

$$
\psi_{3}=\mathbf{T}_{31} \psi_{1} \text {, }
$$

where $\mathbf{T}_{31}=\mathbf{H}_{2}^{-1} \mathbf{K}_{2} \mathbf{K}_{1}^{-1} \mathbf{H}_{1}$ is the transfer matrix from the 1st layer to the 3rd layer.

With regard to 3 rd layer and 4th layer, the following can be smoothly achieved: $\psi_{3}=\psi_{4}$. Similar to the derivation procedure $\mathbf{T}_{31}=\mathbf{H}_{2}^{-1} \mathbf{K}_{2} \mathbf{K}_{1}^{-1} \mathbf{H}_{1}$, according to the interface continuity at $e$ and $f$, one has

$$
\psi_{6}=\mathbf{T}_{64} \psi_{4} .
$$

Similarly, transfer matrix from the 6th layer to the 8 th layer can be obtained as

$$
\psi_{8}=\mathbf{T}_{86} \psi_{6} .
$$

Finally, combining equations (29)-(31) reduces to

$$
\begin{aligned}
\psi_{8} & =\mathbf{T}_{81} \psi_{1} \\
& =\mathbf{T}_{86} \mathbf{T}_{64} \mathbf{T}_{31} \psi_{1},
\end{aligned}
$$

where $\mathbf{T}_{81}=\mathbf{T}_{86} \mathbf{T}_{64} \mathbf{T}_{31}$ is the total transfer matrix from the 1st layer to the 8th layer of the piezoelectric Fibonacci RPCAP.

Here, the inner boundary is loaded with transverse displacement and the outer boundary is set free. Thus, the boundary conditions at the inner edge $r_{1}$ are

$$
\begin{array}{r}
A_{11} J_{0}\left(k_{1} r_{1}\right)+B_{11} Y_{0}\left(k_{1} r_{1}\right)+C_{11} I_{0}\left(k_{1} r_{1}\right)+D_{11} K_{0}\left(k_{1} r_{1}\right)=1, \\
-k_{1} A_{11} J_{1}\left(k_{1} r_{1}\right)-k_{1} B_{11} Y_{1}\left(k_{1} r_{1}\right)+k_{1} C_{11} I_{1}\left(k_{1} r_{1}\right)-k_{1} D_{11} K_{1}\left(k_{1} r_{1}\right)=0 .
\end{array}
$$

The boundary conditions at the outer edge $r_{2}$ are 


$$
\begin{aligned}
& A_{18}\left[\frac{k_{2}}{r_{2}} J_{1}\left(k_{2} r_{2}\right)-\frac{\sigma_{2} k_{2}}{r_{2}} J_{1}\left(k_{2} r_{2}\right)-k_{2}^{2} J_{0}\left(k_{2} r_{2}\right)\right]+B_{18}\left[\frac{k_{2}}{r_{2}} Y_{1}\left(k_{2} r_{2}\right)-\frac{\sigma_{2} k_{2}}{r_{2}} Y_{1}\left(k_{2} r_{2}\right)-k_{2}^{2} Y_{0}\left(k_{2} r_{2}\right)\right] \\
& \quad+C_{18}\left[k_{2}^{2} I_{0}\left(k_{2} r_{2}\right)-\frac{k_{2}}{r_{2}} I_{1}\left(k_{2} r_{2}\right)+\frac{\sigma_{2} k_{2}}{r_{2}} I_{1}\left(k_{2} r_{2}\right)\right]+D_{18}\left[k_{2}^{2} K_{0}\left(k_{2} r_{2}\right)+\frac{k_{2}}{r_{2}} K_{1}\left(k_{2} r_{2}\right)-\frac{\sigma_{2} k_{2}}{r_{2}} K_{1}\left(k_{2} r_{2}\right)\right]=0 \\
& A_{18} k_{2}^{3} J_{1}\left(k_{2} r_{2}\right)+B_{18} k_{2}^{3} Y_{1}\left(k_{2} r_{2}\right)+C_{18} k_{2}^{3} I_{1}\left(k_{2} r_{2}\right)-D_{18} k_{2}^{3} K_{1}\left(k_{2} r_{2}\right)=0
\end{aligned}
$$

Combining equations (33)-(36) yields

$$
\psi_{1}=\left[\begin{array}{llll}
\mathbf{P}_{1} & \mathbf{P}_{2} & \mathbf{P}_{3} & \mathbf{P}_{4}
\end{array}\right]^{-1}\left[\begin{array}{llll}
1 & 0 & 0 & 0
\end{array}\right]^{T}
$$

where

$$
\begin{aligned}
& \mathbf{P}_{1}=\left[J_{0}\left(k_{1} r_{1}\right)-k_{1} J_{1}\left(k_{1} r_{1}\right) \frac{k_{2}\left\{J_{1}\left(k_{2} r_{2}\right)-\sigma_{2} J_{1}\left(k_{2} r_{2}\right)\right\}}{r_{2}}-k_{2}^{2} J_{0}\left(k_{2} r_{2}\right) k_{2}^{3} J_{1}\left(k_{2} r_{2}\right)\right]^{T}, \\
& \mathbf{P}_{2}=\left[Y_{0}\left(k_{1} r_{1}\right)-k_{1} Y_{1}\left(k_{1} r_{1}\right) \frac{k_{2}\left\{Y_{1}\left(k_{2} r_{2}\right)-\sigma_{2} Y_{1}\left(k_{2} r_{2}\right)\right\}}{r_{2}}-k_{2}^{2} Y_{0}\left(k_{2} r_{2}\right) k_{2}^{3} Y_{1}\left(k_{2} r_{2}\right)\right]^{T} \text {, } \\
& \mathbf{P}_{3}=\left[I_{0}\left(k_{1} r_{1}\right) k_{1} I_{1}\left(k_{1} r_{1}\right) \frac{k_{2}\left\{\sigma_{2} I_{1}\left(k_{2} r_{2}\right)-I_{1}\left(k_{2} r_{2}\right)\right\}+r_{2} k_{2}^{2} I_{0}\left(k_{2} r_{2}\right)}{r_{2}} k_{2}^{3} I_{1}\left(k_{2} r_{2}\right)\right]^{T}, \\
& \mathbf{P}_{4}=\left[K_{0}\left(k_{1} r_{1}\right) k_{1} K_{1}\left(k_{1} r_{1}\right) \frac{k_{2}\left\{K_{1}\left(k_{2} r_{2}\right)-\sigma_{2} K_{1}\left(k_{2} r_{2}\right)\right\}}{r_{2}}+k_{2}^{2} K_{0}\left(k_{2} r_{2}\right)-k_{2}^{3} K_{1}\left(k_{2} r_{2}\right)\right]^{T} \text {. }
\end{aligned}
$$

Considering equations (35)-(37), the transverse displacement $W^{I}$ at the outermost layer can be calculated as $W^{I}=\mathbf{T}_{\mathbf{8 1}} \psi_{\mathbf{1 1}}\left[k_{2}^{3} J_{1}\left(k_{2} r_{2}\right) k_{2}^{3} Y_{1}\left(k_{2} r_{2}\right) k_{2}^{3} I_{1}\left(k_{2} r_{2}\right) k_{2}^{3} K_{1}\left(k_{2} r_{2}\right)\right]$.

Thus, the vibration transmissibility can be written as

$$
\mathrm{dB}=20 \log \left(\frac{W^{I}}{1}\right),
$$

where $\mathrm{dB}$ represents the displacement amplitude of the vibration transmissibility picked at the outermost layer boundary $r_{2}$.

\section{Analysis and Discussion}

In this section, transmission response is presented for the piezoelectric Fibonacci RPCAP to obtain the band behaviors of transverse wave. Through adopting MATLAB software, the derived vibration transmissibility $\mathrm{dB}$ and transfer matrix $\mathbf{T}_{81}$ are combined to provide a numerical approach for the transverse wave band analysis of this Fibonacci RPCAP. Here, the radial lengths of the resin and PZT4 are $a_{1}=0.01 \mathrm{~m}$, $a_{2}=0.01 \mathrm{~m}$. The inner radius is $r_{1}=0.005 \mathrm{~m}$. The thickness is $h=0.001 \mathrm{~m}$. Material parameters are given as follows:

Resin: modulus $E_{1}=0.435 \times 10^{10} \mathrm{~Pa}$, density $\rho_{1}=$ $1180 \mathrm{~kg} / \mathrm{m}^{3}$, and Poisson's ratio $\sigma_{1}=0.3679$.
PZT4: elastic coefficient $s_{11}^{D}=10.9 \times 10^{-12} \mathrm{~m}^{2} / \mathrm{N}$, coupling coefficient $k_{p}=0.58$, piezoelectric constant $\mathrm{g}_{31}=-11.1 \times 10^{-3} \mathrm{~V} \cdot \mathrm{m} / \mathrm{N}$, dielectric constant $\beta_{33}^{T}=$ $1300, \bar{\beta}_{33}=1959$, modulus $E_{2}=9.17 \times 10^{10} \mathrm{~Pa}$, density $\rho_{2}=7500 \mathrm{~kg} / \mathrm{m}^{3}$, and Poisson's ratio $\sigma_{2}=0.33$.

Like other types of Cartesian crystals, it is impossible to deal with infinite PC structure. Here, a piezoelectric Fibonacci RPCAP consisting of eight layers along the radial direction, corresponding to nonperiodic model ABAA$\mathrm{BABA}$, is considered to investigate the stop band behaviors. Afterwards, numerical examples for the three cases: single resin, resin/Al, and resin/PZT4, are compared to reveal the unique dynamic characteristics of Fibonacci RPCAP, as depicted in Figure 2. It can be found that the transverse vibration attenuates nearly -10 decibel while transverse wave propagates in a single resin. Actually, generally speaking, there is no vibration attenuation for the single material case. Therefore, the vibration attenuation of -10 decibel is considered to be caused by the radial wave front [12]. Compared with the single resin case, a newly emerging vibration attenuation band can be captured for the resin/Al case while the radial Fibonacci order is introduced into the RPCAP model. This vibration attenuation region corresponds to stop bands III $(9.4 \mathrm{kHz}-19.2 \mathrm{kHz})$ and IV $(2.56 \mathrm{kHz}-3.75 \mathrm{kHz})$ which attenuate about -30 decibel. Obviously, vibration attenuation of transverse wave propagating in Fibonacci RPCAP model is much more effective 


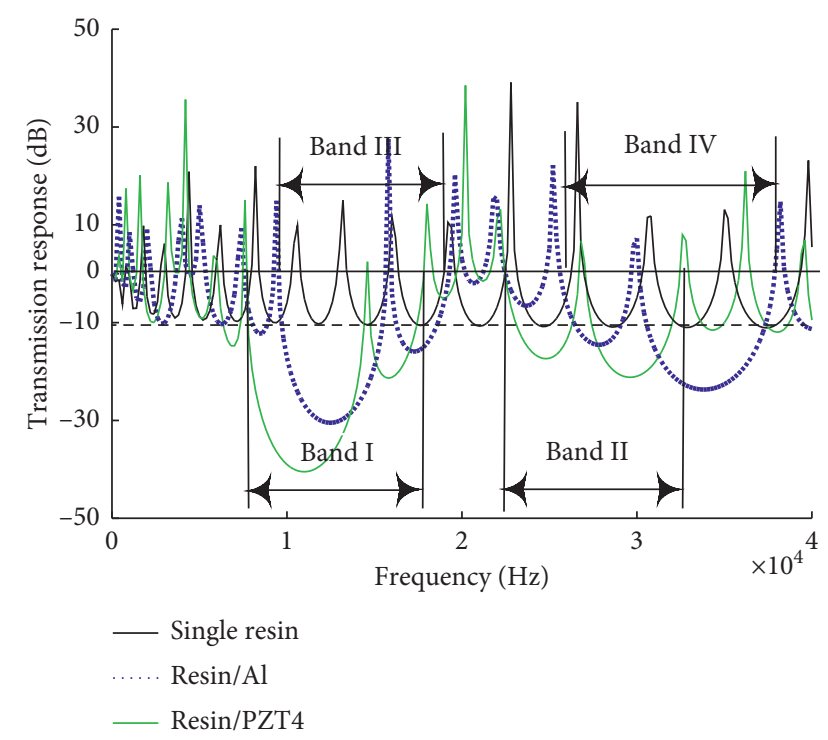

Figure 2: Theoretical curves.

than that in a single resin case, which is caused by the radial periodicity.

Additionally, the transmission response curves present significant transverse wave stop bands, I $(7.6 \mathrm{kHz}-17.8 \mathrm{kHz})$ and II $(22.4 \mathrm{kHz}-32.5 \mathrm{kHz})$, attenuated about -40 decibel from which one can arrive at the conclusion that vibration attenuation in resin/PZT4 RPCAP is much more heavy than that in the resin/Al case. After introducing piezoelectric material PZT4 into Fibonacci RPCAP, the location of stop band moves toward the low frequency gradually. In addition to the vibration attenuation caused by the expansion of wave front, the attenuation below the dashed lines shown in Figure 2 is caused by the Fibonacci order of RPCAP. Numerical results also show that there are many other small attenuation stop bands for this Fibonacci RPCAP.

In order to illustrate the validity of theoretical calculation, finite element simulation is conducted with ANSYS 14.0 software to show the location and width of the stop bands. In this case, the resin material is meshed with solid45 element while solid226 element is employed to mesh piezoelectric material. The total number of elements is 3145 . Transverse displacement is loaded on the inner radial surface of the Fibonacci RPCAP, and then displacement at the outmost radial surface is picked. Here, harmonic response analysis is carried out to perform the stop bands. Figure 3 shows the simulated transmission response curves from which we can see that the location and width of transverse bands coincide with theoretical results depicted in Figure 2 very well. Therefore, the simulated curves verify the correctness of theoretical results.

In order to better show the stop band behaviors after introducing Fibonacci order, transmission response curves of transverse wave propagating in piezoelectric Fibonacci RPCAP model and periodic RPCAP model are plotted as described in Figure 4. The two curves shown in Figure 4 are depicted for comparison. From these curves, we can see that

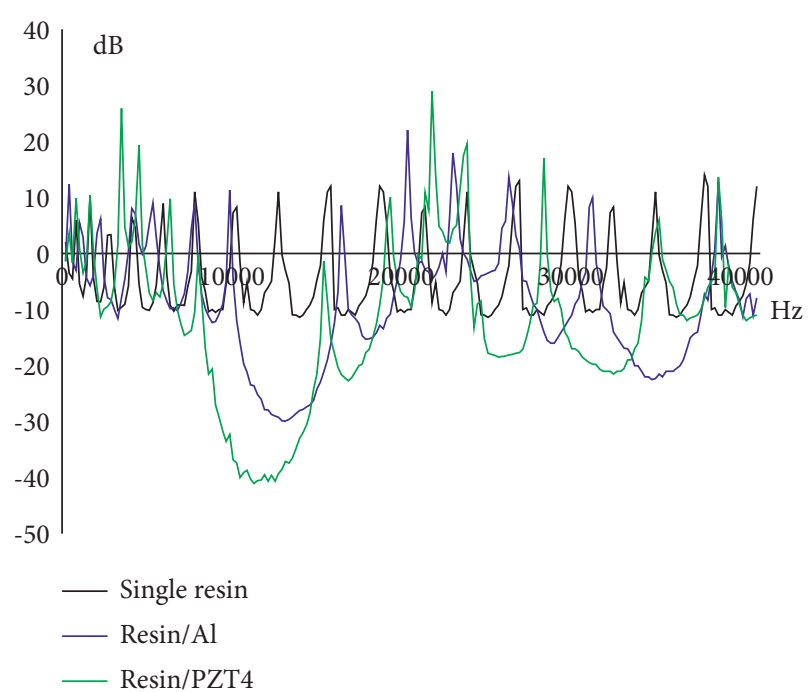

Figure 3: FEM curves.

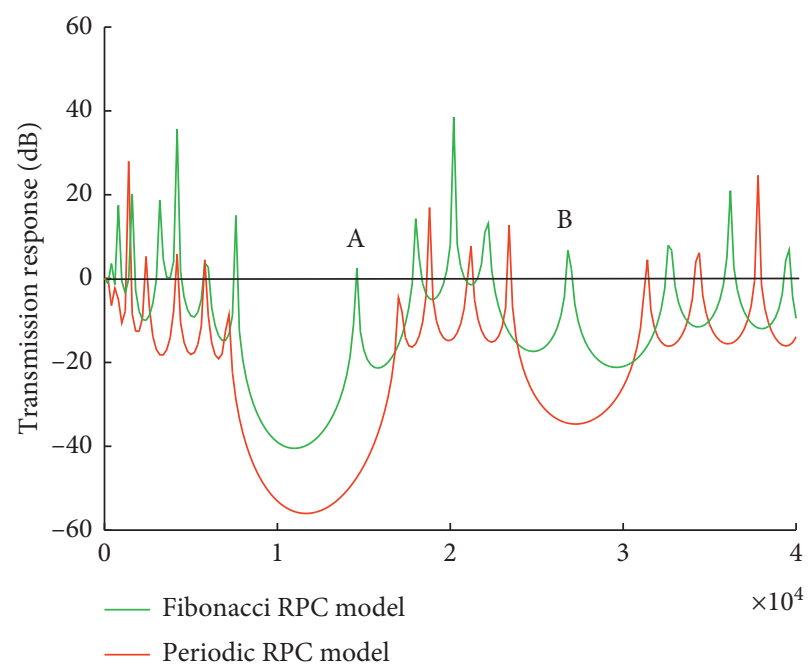

FIgURE 4: Theoretical curves.

compared with the periodic RPCAP model, the Fibonacci RPCAP model presents two newly emerging resonance frequencies corresponding to points $A$ and $B$. We consider that the two resonance points are caused by the radial Fibonacci order. In other words, the radial Fibonacci order breaks the previous radial periodicity. The results also show that after introducing radial Fibonacci order, the location and width of stop bands move toward high frequency. Additionally, finite element simulation results depicted in Figure 5 verify the correctness of theoretical curves corresponding to Figure 4.

\section{Influence Factors}

Structural size, material characteristic, and piezoelectric performance are the main parameters that affect the vibration transmission response of composite structures. Also, these parameters are commonly employed to analyze the 


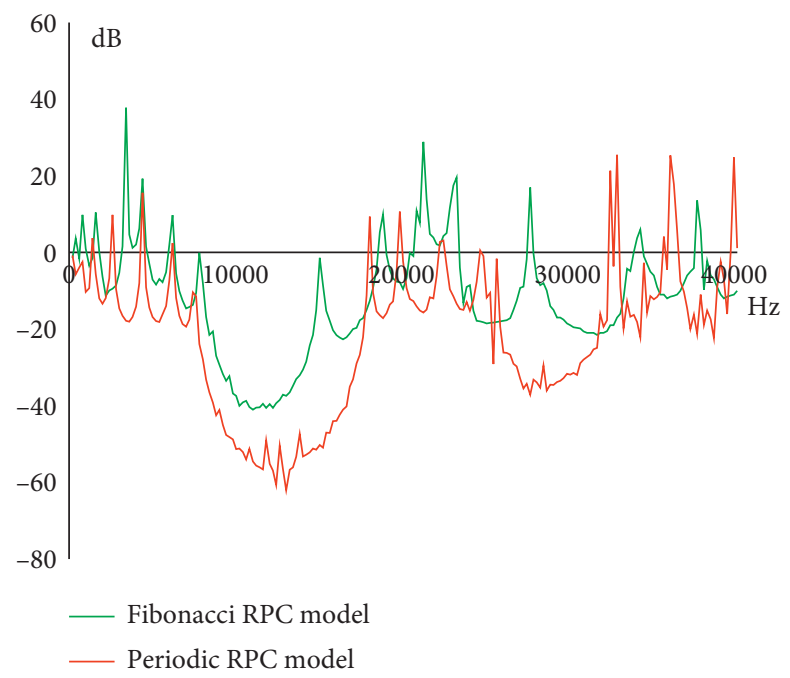

Figure 5: FEM curves.

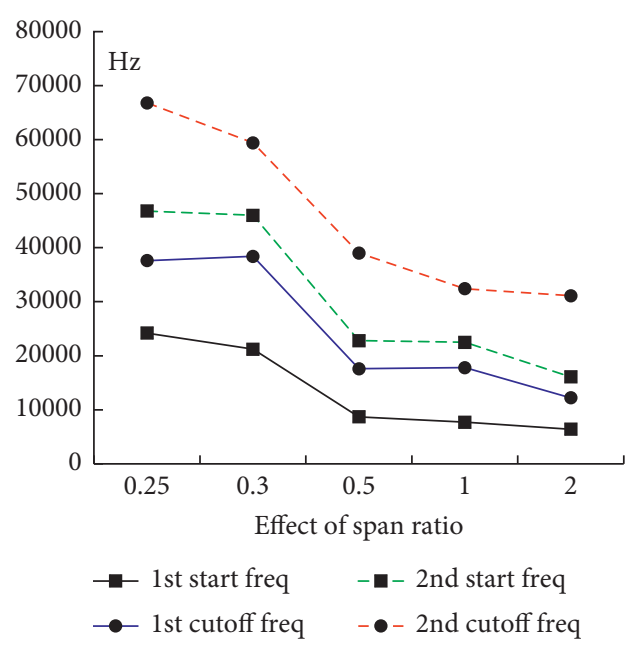

(a)

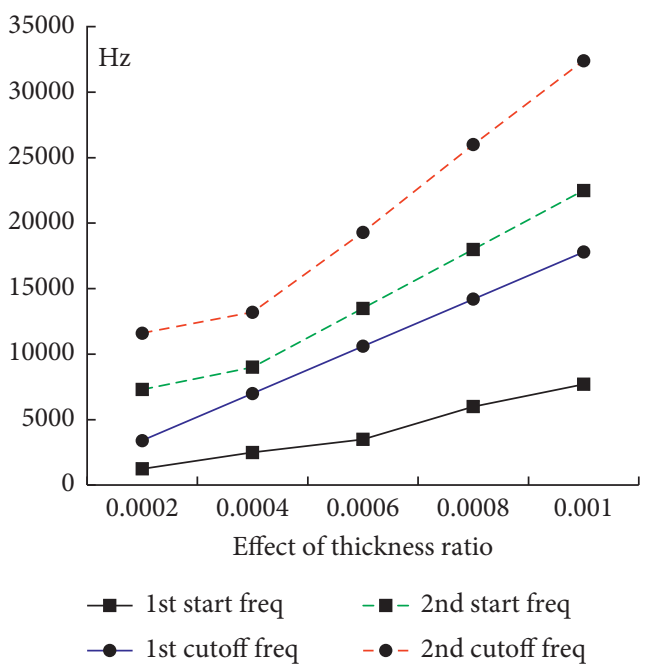

(b)

Figure 6: Effect of span ratio and thickness ratio on stop bands: (a) span ratio; (b) thickness ratio.

stop band behaviors of elastic wave. Accordingly, in this section, the effects of these parameters on the stop bands are discussed in detail.

4.1. Structural Parameters. With regard to piezoelectric Fibonacci RPCAP, the effect of span ratio on the transverse wave stop bands is studied. Keeping $a=a_{1}+a_{2}$ and other structural parameters unchanged, we only change the radial span ratio $a_{1} / a_{2}$. Figure $6(\mathrm{a})$ describes the effect of span ratio on the start and cutoff frequencies for the first and second stop bands. It can be seen that with the span ratio increasing, the start and cutoff frequencies decrease gradually. Similarly, adopting a similar approach, Figure 6(b) presents the effect of thickness ratio on the start and cutoff frequencies for the first and second bands. With the thickness ratio increasing, the start and cutoff frequencies increase rapidly, and simultaneously the bandwidths become larger.

Effect of inner radius magnitude on the transverse wave stop bands is depicted in Figure 7. We can see from Figure 7 that with the inner radius increasing, transmission response for this Fibonacci RPCAP changes very small. In other words, the location and width of the stop bands almost have no change, but the stop bands attenuate heavily.

4.2. Material Parameters. Similar to the study on the effect of structure parameters, Figure 8 shows the effect of modulus and density on the transverse wave stop bands. Keeping other parameters unchanged, here the density is selected as a variable parameter. Density of materials are selected as Plexiglas, Al, Titanium alloy, PZT4, and Plumbum. It can be 


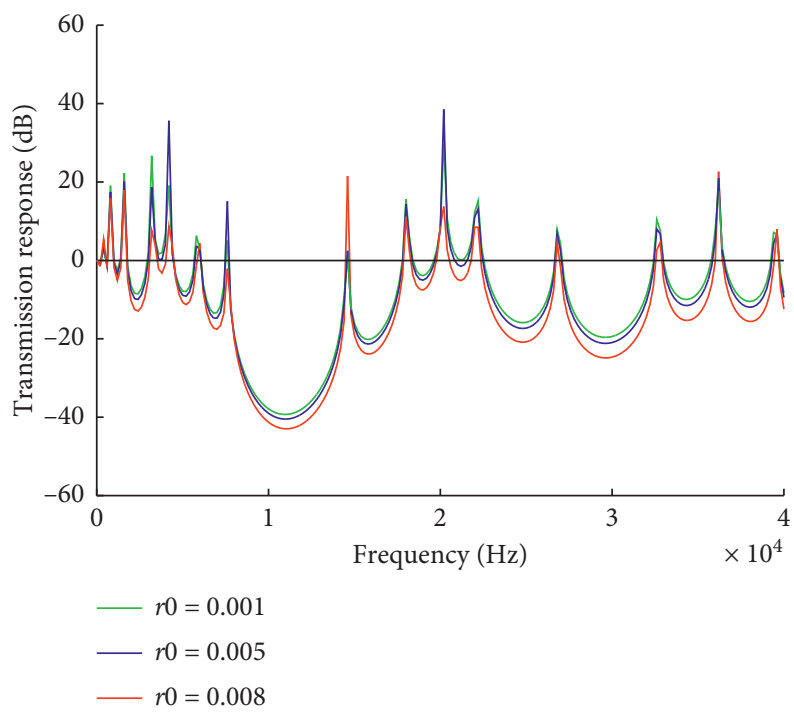

FIGURE 7: Effect of inner radius on stop bands.

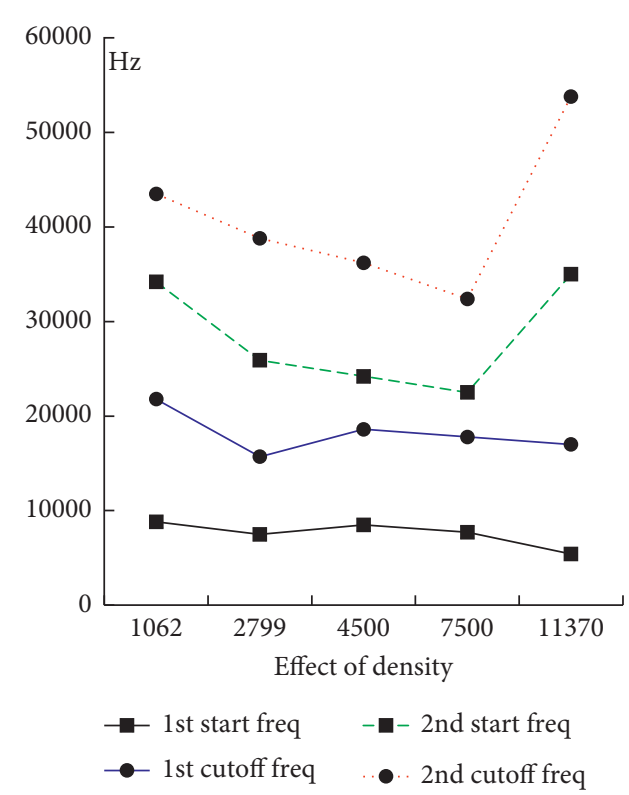

(a)

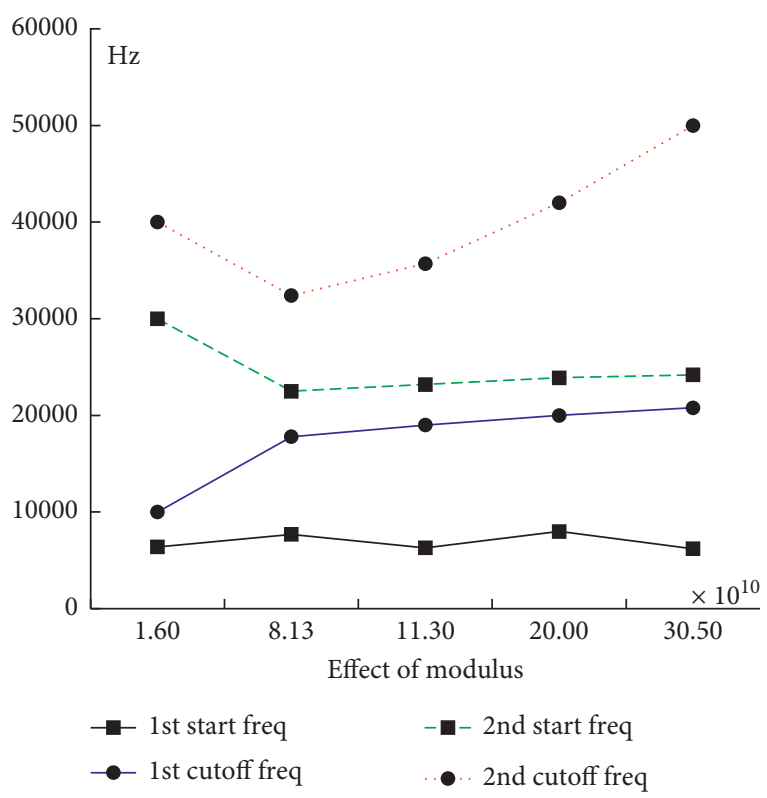

(b)

FIGURE 8: Effect of modulus and density on stop bands: (a) effect of density; (b) effect of modulus.

seen from Figure 8(a) that with the density increasing, the start and cutoff frequencies of first transverse wave band reduce gradually, but the bandwidths almost have no change. Start and cutoff frequencies of the second transverse wave stop band become smaller at first and then gradually become larger. Figure $8(\mathrm{~b})$ shows the effect of modulus on the transverse wave stop bands, illustrating that the modulus can significantly affect these bands. Both start frequencies of the first and second stop bands reduce, and both cutoff frequencies increase gradually with the modulus increasing. Meanwhile, these bandwidths become wider.
4.3. Piezoelectric Parameters. In addition to the effect of structural and material parameters, piezoelectric parameters can also affect the stop band. In this section, the piezoelectric polar direction, electrical open circuit case, and electrical short circuit case are considered here as example to reveal the variation rule of the stop bands.

Bending stiffness of piezoelectric material PZT4 for the electrical short circuit case $D^{E}$ and electrical open circuit case $D^{D}$ has been derived in equations (21) and (22). Adopting MATLAB software to program and substituting $D^{E}, D^{D}$ into total transfer matrix equation (32), the 


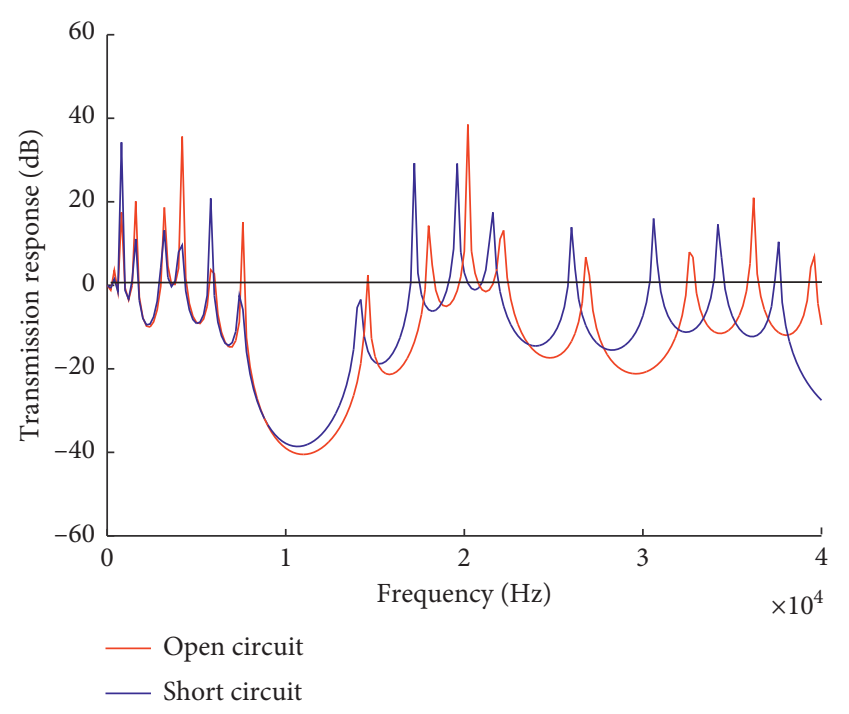

FIgURE 9: Band curves for open and short circuit.

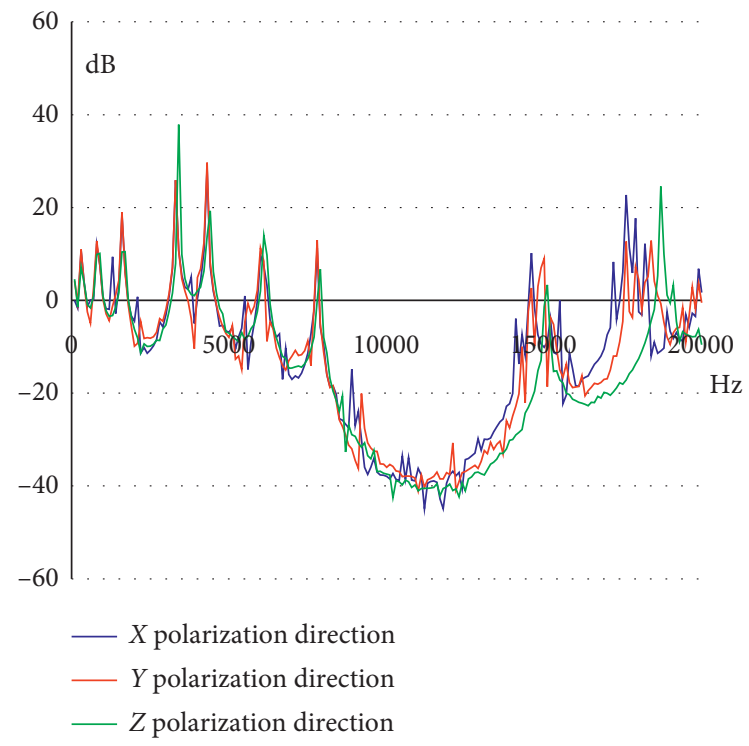

Figure 10: Effect of polar direction.

transmission response curves of piezoelectric Fibonacci RPCAP are plotted as shown in Figure 9. These curves show that electrical short circuit case and open circuit case can significantly affect the transverse wave stop bands. Also, the locations of transverse wave stop bands move toward the low frequency. The above phenomenon also indicates that the bending stiffness of piezoelectric material PZT4 is changed due to the piezoelectric effect. In other words, magnitudes of bending stiffness are determined by the electrical short circuit case and open circuit case, leading to the variation of band location and width.

Adopting finite element simulation approach, piezoelectric material PZT4 is selected along the $X, Y$, and $Z$ polarization directions in cylindrical coordinates. Figure 10 shows the effect of polarization direction on the transmission response of transverse wave for the Fibonacci RPCAP.
It can be seen from Figure 10 that the stop bands attenuate significantly in the high-frequency region, but it is not obvious in the low-frequency region. This phenomenon indicates that polarization direction is also a main reason causing the stop band variation.

In summary, the above transmission response studies indicate that structural parameters, material parameters, and piezoelectric parameters for the Fibonacci RPCAP can significantly affect the transverse wave stop bands. Through properly designing these parameters, one can achieve large and wide elastic wave stop bands for engineering vibration reduction.

\section{Conclusion}

This paper presents a piezoelectric Fibonacci RPCAP model to obtain the transverse wave stop bands. Firstly, based on the solutions of homogeneous material equation and piezoelectric governing equation, expressions of rotational angle, shear force, and bending moment are derived. Then, these parameters in cylindrical coordinates are combined to derive the transfer matrix. The transverse wave stop band behaviors for the Fibonacci RPCAP model are analyzed. Finite element simulation is conducted here with ANSYS 14.0 software to verify the location and width of stop bands.

A further analysis for the influence of structural parameters and material parameters on the stop band is discussed. It can be found that these parameters for the Fibonacci RPCAP can significantly affect the transverse wave stop bands. Additionally, the results also show that piezoelectric performances such as electrical short circuit, electrical open circuit, and PZT4 polarization direction are also the main reason leading to the stop band variation of transverse wave.

Finally, the stop band behaviors of transverse wave are investigated in detail. This work is of great significance to block the wave propagation, especially for those torsional rotor systems coupled with transverse vibration.

\section{Data Availability}

The data used to support the findings of this study are included within the article.

\section{Conflicts of Interest}

The authors declare that there are no conflicts of interest regarding the publication of this paper.

\section{Acknowledgments}

This study was supported by the Fundamental Research Funds for the Central Universities (grant no. 2572020BG01), National Key R\&D Program of China (grant no. 2020YFB2006400), and Major Science and Technology Projects of Heilongjiang Province (grant no. 2020ZX03A03). The authors wish to thank the School of Mechatronics Engineering, Harbin Institute of Technology, Harbin, China, for providing technical support. 


\section{References}

[1] S. Asiri, A. Baz, and D. Pines, "Active periodic struts for a gearbox support system," Smart Materials and Structures, vol. 15, no. 6, pp. 1707-1714, 2006.

[2] D. Wang, S. Zhijun, and L. Wei, C. Meilong, L. Siyuan, and L. Shidan, In-plane vibration analysis of phononic crystal curved beams," Noise Control Engineering Journal, vol. 64, no. 5, pp. 658-667, 2016.

[3] D. Qian, J. Wu, and F. He, "Electro-mechanical coupling band gaps of a piezoelectric phononic crystal Timoshenko nanobeam with surface effects," Ultrasonics, vol. 109, Article ID 106225, 2021.

[4] L. Yao, D. Zhang, K. Xu, L. Dong, and X. Chen, "Topological phononic crystal plates with locally resonant elastic wave systems," Applied Acoustics, vol. 177, Article ID 107931, 2021.

[5] D. Yu, J. Wen, H. Zhao, Y. Liu, and X. Wen, "Vibration reduction by using the idea of phononic crystals in a pipeconveying fluid," Journal of Sound and Vibration, vol. 318, no. 1-2, pp. 193-205, 2008.

[6] M. Mazzotti, M. Miniaci, and I. Bartoli, "Band structure analysis of leaky Bloch waves in 2D phononic crystal plates," Ultrasonics, vol. 74, pp. 140-143, 2017.

[7] P. Chen, Y.-Z. Wang, and Y.-S. Wang, "Active control of flexural waves in a phononic crystal beam with staggered periodic properties," Wave Motion, vol. 93, Article ID 102481, 2020.

[8] A. M. Goto, E. D. Nóbrega, F. N. Pereira, and J. M. C. Dos Santos, "Numerical and experimental investigation of phononic crystals via wave-based higher-order rod models," International Journal of Mechanical Sciences, vol. 181, Article ID 105776, 2020.

[9] A. Zak, M. Krawczuk, G. Redlarski et al., "A three-dimensional periodic beam for vibroacoustic isolation purposes," Mechanical Systems and Signal Processing, vol. 130, pp. 524544, 2019.

[10] X. Guo, P. Wei, and L. Li, "Dispersion relations of elastic waves in one-dimensional piezoelectric phononic crystal with mechanically and dielectrically imperfect interfaces," $M e$ chanics of Materials, vol. 93, pp. 168-183, 2016.

[11] H. S. Shu, L. Q. Dong, S. D. Li et al., "Propagation of torsional wave in a thin annular plate of generalized phononic crystals," Journal of Applied Physics D, vol. 47, pp. 1-11, 2014.

[12] H. Shu, W. Liu, S. Li et al., "Research on flexural wave band gap of a thin circular plate of piezoelectric radial phononic crystals," Journal of Vibration and Control, vol. 22, no. 7, pp. 1777-1789, 2016.

[13] Z. Chai, D. Wang, W. Liu, and D. Kong, "Torsional wave propagation in a piezoelectric radial phononic crystals," Noise Control Engineering Journal, vol. 64, no. 1, pp. 75-84, 2016.

[14] Z. Xu, F. Wu, and Z. Guo, "Low frequency phononic band structures in two-dimensional arc-shaped phononic crystals," Physics Letters A, vol. 376, no. 33, pp. 2256-2263, 2012.

[15] D. Torrent and J. Sánchez-Dehesa, "Acoustic resonances in two-dimensional radial sonic crystal shells," New Journal of Physics, vol. 12, no. 7, Article ID 073034, 2010.

[16] D. Torrent and J. Sánchez-Dehesa, "Radial wave crystals: radially periodic structures from anisotropic metamaterials for engineering acoustic or electromagnetic waves," Physical Review Letters, vol. 103, Article ID 064301, 2009.

[17] J. Carbonell, D. Torrent, and J. Sanchez-Dehesa, "Radial photonic crystal shells and their application as resonant and radiating elements," IEEE Transactions on Antennas and Propagation, vol. 61, no. 2, pp. 755-767, 2013.
[18] H. S. Shu, L. Zhao, X. N. Shi, and W. Liu, "Torsional wave propagation in a circular plate of piezoelectric radial phononic crystals," Journal of Applied Physics, vol. 118, no. 2, Article ID 184904, 2015. 